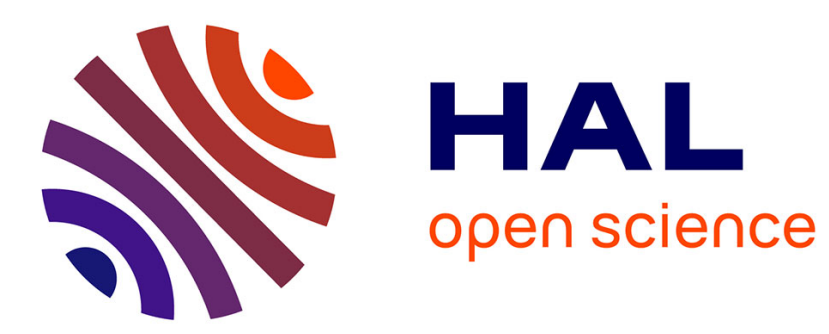

\title{
AMM : entrectinib et larotrectinib - cancers avec fusion NTRK
}

\author{
Matthieu Delaye, Manuel Rodrigues
}

\section{To cite this version:}

Matthieu Delaye, Manuel Rodrigues. AMM : entrectinib et larotrectinib - cancers avec fusion NTRK. Bulletin du Cancer, 2020, 107 (11), pp.1085-1086. 10.1016/j.bulcan.2020.09.009 . hal03134384

\section{HAL Id: hal-03134384 https://hal.sorbonne-universite.fr/hal-03134384}

Submitted on 8 Feb 2021

HAL is a multi-disciplinary open access archive for the deposit and dissemination of scientific research documents, whether they are published or not. The documents may come from teaching and research institutions in France or abroad, or from public or private research centers.
L'archive ouverte pluridisciplinaire HAL, est destinée au dépôt et à la diffusion de documents scientifiques de niveau recherche, publiés ou non, émanant des établissements d'enseignement et de recherche français ou étrangers, des laboratoires publics ou privés. 
Nouvelle AMM: entrectinib et larotrectinib - cancers avec fusion NTRK

Matthieu Delaye ${ }^{1,2}$, Manuel Rodrigues ${ }^{3,4}$

1. Service d'oncologie médicale et de thérapie cellulaire, hôpital Tenon, 4 rue de la Chine, 75020, Paris, France

2. Association pour l'enseignement et la recherche des internes en oncologie (AERIO), 149, avenue du Maine, 75014 Paris, France.

3. Institut Curie, PSL Research University, Departement d'Oncologie Médicale, 26, rue d'Ulm, 75005 Paris, France.

4. Institut Curie, PSL Research University, Inserm U830, 26, rue d'Ulm, 75005 Paris, France.

\section{Correspondant :}

Manuel Rodrigues, Institut Curie, Département d'Oncologie Médicale, 26, rue d'Ulm, 75248 Paris Cedex 05, France

tel 0144324680

Fax 0153104041

Mots clés : Thérapie Ciblée, NTRK, Tissu-Agnostique

Keywords: Targeted Therapy, NRTK, tissue-agnostic 
A l'heure de la génomique et des thérapies ciblées, des progrès considérables ont été faits avec la découverte de nombreuses anomalies ciblables en thérapeutique dans différents cancers. L'idée que des anomalies communes à plusieurs cancers puissent être ciblées par un seul et même traitement, un traitement dit « tissu-agnostique », est séduisante mais, jusqu'il y a peu, aucune molécule n'avait réuni des éléments suffisants pour obtenir une autorisation de mise sur le marché (AMM) européen. Les gènes NTRK1,2 et 3 (pour Neurotrophic Receptor Tyrosine Kinase) codent pour les récepteurs TRKA, B et C (pour Tropomyosin Receptor Kinase). En cancérologie, des transcrits de fusion impliquant le domaine kinase des TRK ont été mis en évidence dans différents cancers pédiatriques et de l'adulte, les plus fréquents étant les tumeurs des glandes salivaires, les sarcomes, les tumeurs gliales ou les carcinomes thyroïdiens [1]. Ces fusions entraînent la sur-expression de protéines chimériques et finalement l'activation constitutionnelle de voies de signalisation pro-tumorales. Elles seraient présentes dans près de $1 \%$ des cancers solides [2]. Plusieurs inhibiteurs des TRK ont été développés, parmi lesquels le larotrectinib (Loxo Oncology) et l'entrectinib (Genentech). Trois essais de phase 1 et 2 (LOXO-TRK-14001, SCOUT, et NAVIGATE) ont permis au larotrectinib d'obtenir une approbation de la FDA (Food and Drug Administration) fin 2018 puis de l'EMA (European Medicines Agency) en 2019. A présent c'est l'entrectinib qui obtient ces agréments suite à la publication de I'analyse combinée des essais ALKA-372-001, STARTRK-1 et STARTRK-2.

Pour approuver le larotrectinib, la FDA et l'EMA se sont basées sur les données préliminaires de 3 essais en cours de phase 1, 1/2 et 2 : LOXO-TRK-14001, SCOUT et NAVIGATE, dont l'analyse combinée a été publiée dans le New England Journal of Medicine. [3] Elle porte sur 55 patients, enfants et adultes, pris en charge pour différents cancers localement avancés (10 patients) ou métastatiques (45 patients), les plus représentés étant les tumeurs des glandes salivaires (12 patients), les sarcomes des tissus mous (11 patients) et les fibrosarcomes de l'enfant (7 patients). Un seul patient avait des lésions cérébrales. La présence d'une fusion NTRK était mise en évidence par séquençage pantranscriptomique de l'ARN messager (RNAseq) ou par FISH (Fluorescent Hybridization In Situ). Les patients recevaient soit une dose croissante de larotrectinib, s'ils étaient en phase 1 , soit une dose de 
$100 \mathrm{mg}$ deux fois par jour s'ils étaient en phase 2. Les résultats sont à première vue prometteurs. Le taux de réponse global était de $75 \%$ incluant $22 \%$ de réponses complètes et $53 \%$ de réponses partielles. La durée médiane de réponse et la survie sans progression (PFS) n'étaient pas atteintes avec un suivi médian de 9,9 mois. Les données de tolérance sont satisfaisantes: les effets secondaires principaux étaient la cytolyse hépatique ( $42 \%$ dont $7 \%$ de grade $\geq 3$ ), I'asthénie ( $36 \%$ dont $2 \%$ de grade $\geq 3$ ), les vomissements ( $33 \%$ dont $0 \%$ de grade $\geq 3$ ) et les cytopénies. Cette présomption d'activité et le profil de tolérance favorable ont permis d'abord une autorisation temporaire d'utilisation (ATU) de cohorte puis une autorisation de mise sur le marché. Ces résultats ont été récemment complétés par les données de plus de patients issus de ces essais. [4] Chez ces 159 patients (incluant les 55 cités précédemment) le taux de réponse objective était de $79 \%$ dont $16 \%$ de réponses complètes, confirmant les résultats intéressants du larotrectinib.

Les données cliniques qui concernent l'entrectinib sont également issues d'essais de phase 1 et 2. Leur analyse combinée a été publiée récemment. [5] Elle reprend les données de 54 patients inclus dans les essais ALKA-372-001, STARTRK-1 et STARTRK-2, traités pour différents primitifs métastatiques ou localement avancés, incluant 12 patients avec des lésions cérébrales. Ils étaient traités par 600 mg d'entrectinib une fois par jour. Les résultats sont intéressants également avec 31 réponses objectives (57\%) dont 4 réponses complètes (7\%). Chez les patients avec métastases cérébrales : 6 présentaient une réponse objective (50\%). La tolérance était satisfaisante également avec peu d'effets secondaires de haut grade, et principalement une toxicité hématologique, une asthénie et des troubles digestifs.

L'AMM du larotrectinib en Europe est en monothérapie dans le traitement des patients adultes et pédiatriques à partir d'un mois, atteints de tumeurs solides localement avancées ou métastatiques présentant une fusion NTRK, réfractaires aux traitements standards ou en l'absence d'alternative thérapeutique appropriée. Les indications proposées par le CHMP (Committee for Medicinal Products 
for Human Use) de l'EMA pour l'entrectinib sont similaires avec une limitation toutefois aux enfants de plus de 12 ans.

L'arrivée de ces traitements soulève un certain nombre de questionnements. La première question est celle de la mise en évidence de l’anomalie moléculaire ciblée dans la pratique. Un algorithme pour le diagnostic de ces translocations reste à mettre en place, en utilisant d'abord une méthode peu coûteuse et sensible comme l'immunohistochimie, puis en ne réalisant une FISH ou un RNAseq que chez les patients positifs. Dans les types histologiques présentant fréquemment ces translocations (tumeurs myofibroblastiques ou des glandes salivaires par exemple), on irait directement au test le plus spécifique (FISH ou séquençage). Toutefois, le RNAseq se démocratise, la technique se simplifie, le processus est plus court, le coût est en baisse, ce qui le rendra accessible à tous nos patients en rechute dans les prochaines années. La problématique principale est l'absence de bras comparateur dans les essais, chez des patients parfois peu ou non traités de leur cancer, ce qui ne permet pas de conclure à la supériorité des inhibiteurs de TRK sur les traitements standards dans certaines indications. Notons toutefois que l'amplitude de l'effet thérapeutique est importante, y compris dans des cancers avec peu d'options thérapeutiques, trop rares pour permettre la réalisation d'essais cliniques de grande amplitude. Cela pose néanmoins problème dans l'évaluation de l'amélioration du service médical rendu. Du fait de ces limitations, un certain nombre d'Etats n’ont pas accordé de remboursement au larotrectinib, et adopteront probablement la même attitude avec l'entrectinib. En France, la commission de transparence de la HAS s'est positionnée pour une limitation du remboursement aux patients pédiatriques ayant un fibrosarcome infantile ou un autre sarcome des tissus mous, remplissant par ailleurs les critères de l'AMM. Ces réflexions et décisions seront particulièrement instructives pour les patients, les cliniciens et les industriels. II s'agit des premières AMM tissu-agnostiques en Europe et ces décisions guideront les prochains développements de traitements tissu-agnostiques, en particulier des inhibiteurs NTRK de seconde génération tels que le LOXO-195 et le TPX-0005. 


\section{References:}

[1] Ouali K, Pellat A, Cohen R, Svrcek M, Penault-Llorca F, et al. NTRK Fusions: A new way of treatment for gastro-intestinal tumor?. Bull Cancer. avr 2020;107(4):447 -57.

[2] Stransky N, Cerami E, Schalm S, Kim JL, Lengauer C. The landscape of kinase fusions in cancer. Nature Communications. 10 sept 2014;5(1):4846.

[3] Drilon A, Laetsch TW, Kummar S, DuBois SG, Lassen UN, et al. Efficacy of Larotrectinib in TRK Fusion-Positive Cancers in Adults and Children. N Engl J Med. 22 févr 2018;378(8):731 -9.

[4] Hong DS, DuBois SG, Kummar S, Farago AF, Albert CM, et al. Larotrectinib in patients with TRK fusion-positive solid tumours: a pooled analysis of three phase $1 / 2$ clinical trials. The Lancet Oncology. 1 avr 2020;21(4):531- 40.

[5] Doebele RC, Drilon A, Paz-Ares L, Siena S, Shaw AT, et al. Entrectinib in patients with advanced or metastatic NTRK fusion-positive solid tumours: integrated analysis of three phase 1-2 trials. The Lancet Oncology. 1 févr 2020;21(2):271- 82. 